\title{
Dinámica de una partícula magnética bajo la influencia de campos oscilatorios
}

\author{
Dynamics of a magnetic particle under the influence of oscillatory fields
}

\author{
Ana M. Cabanas ${ }^{1} \quad$ David Laroze $^{1}$
}

Recibido 3 de julio de 2014, aceptado 1 de septiembre de 2014

Received: July 3, 2014 Accepted: September 1, 2014

\begin{abstract}
RESUMEN
En este artículo caracterizamos la dinámica de la magnetización de una partícula magnética anisotrópica bajo la acción de campos magnéticos externos. La ecuación de movimiento en estudio es la ecuación de Landau-Lifshitz-Gilbert. La anisotropía del sistema se asume uni-axial, mientras que los campos magnéticos externos están compuestos de un término constante y uno dependiente del tiempo, perpendiculares entre sí. Para la componente dependiente del tiempo, se analiza tanto el caso de un forzamiento periódico como uno cuasiperiódico. Se utilizan diferentes indicadores para detectar los distintos comportamientos dinámicos, tal como exponentes de Lyapunov y espectros de Fourier. Tanto para forzamientos periódicos como cuasiperiódicos se muestra que el sistema exhibe tanto estados caóticos como regulares dependiendo de los parámetros. Se hace un análisis intensivo desde el punto paramétrico, ya que en el cálculo del máximo exponente de Lyapunov se varían simultáneamente dos parámetros. En el último caso otros estados llamados "atractores extraños no caóticos" también han sido encontrados.
\end{abstract}

Palabras clave: Dinámica de espines, caos, atractores extraños no caóticos.

\section{ABSTRACT}

In this work, we characterize the magnetization dynamics of an anisotropic magnetic particle under the presence of external magnetic fields. The temporal evolution is described by the Landau-Lifshitz-Gilbert equation. The anisotropy is assumed uniaxial, whereas the external magnetic fields are composed by a homogenous and a time-dependent one. We analyze the case of a periodic and a quasi-periodic time driven dependent field. Different indicators are used to detect the dynamical behaviors, such as Lyapunov exponent and Fourier power spectrum. We show that for both periodic and quasi-periodic driven fields the system exhibits chaotic and regular states. We perform an intensive analysis from the parametric point of view, since we vary simultaneously two parameters when the largest Lyapunov exponent is calculated. Finally, we found that there are other states called strange non-chaotic attractors.

Keywords: Spin dynamics, chaos, strange nonchaotic attractors.

\section{INTRODUCCIÓN}

La dinámica de la magnetización ha sido estudiada ampliamente debido a sus implicaciones en posibles aplicaciones tecnológicas, por ejemplo, en espintrónica [1], magnetismo molecular [2], bio-magnetismo para usos medicinales [3] o en magnónica [4], solo por nombrar algunos. Además, varios enfoques sobre el aspecto no-lineal del comportamiento dinámico de partículas magnéticas clásicas han sido un tema de investigación recurrente en las últimas décadas.

\footnotetext{
1 Instituto de Alta Investigación. Universidad de Tarapacá. Casilla 7D. C/ Antofagasta 1520. Arica, Chile. E-mail: ana.cabanas.plana@gmail.com; dlarozen@uta.cl
} 
El estado del arte se puede encontrar en las referencias [5-6].

Las aproximaciones más usadas para describir las ecuaciones de movimientos son las ecuaciones de Landau -Lifshitz o Landau-Lifshitz-Gilbert (LLG) [6], esta última será utilizada en este artículo. En este contexto se ha estudiado la dinámica de sistemas magnéticos con diferentes configuraciones tanto de campos externos, como de ejes de anisotropía [7-18], dando lugar a fenómenos como el caos; de hecho estos fenómenos caóticos han sido medidos experimentalmente [7-10]. Sin embargo, no han mostrado una única ruta al caos, por lo que el estudio teórico sobre estos sistemas puede ayudar a dilucidar cuáles son las diferentes rutas al caos en sistemas magnéticos.

Por otra parte, los sistemas forzados cuasiperiódicamente han sido estudiados teórica y experimentalmente en diferentes ramas de la física en las últimas décadas [19-23]. Este tipo de forzamiento es la extensión natural de una única frecuencia de forzamiento, y la razón por la cual uno puede esperar un comportamiento mucho más complejo. De hecho, los conjuntos invariantes más simples son en forma de toros [20]. Más aún, hay comúnmente otros tipos de atractores, llamados atractores extraños no-caóticos, donde "extraño" implica que la topología atractor es complicada y no lisa; y donde "no-caótico" indica que no tiene dependencia sensible de las condiciones iniciales [24].

El objetivo de este trabajo es investigar el comportamiento dinámico de una partícula magnética anisotrópica en presencia de un campo magnético externo aplicado dependiente del tiempo. En particular, nos focalizaremos tanto en campos magnéticos periódicos como cuasiperiódicos, caracterizando la dinámica con diferentes indicadores como son el espectro de Fourier, diagramas de bifurcación y exponentes de Lyapunov. Desde el punto de vista numérico, para el cómputo de los exponentes de Lyapunov se hacen cálculos intensivos ya que se varían dos parámetros simultáneamente, creando diagramas de fases completos [25]. Este trabajo es una continuación y extensión de la referencia [23], donde se estudió el caso puramente cuasiperiódico. En el presente artículo se analizan y comparan las diferencias de tener forzamientos periódicos y cuasiperiódicos. Además, se hace un análisis más detallado de los atractores extraños no caóticos.

Finalmente, haremos un análisis comparativo del máximo exponente de Lyapunov para ambos tipos de forzamiento. El manuscrito está ordenado de la siguiente forma: En la sección dos se expone el modelo teórico. En la sección tres se muestran los resultados numéricos y se hace un análisis de ellos. Por último, en la última sección se exponen las conclusiones.

\section{MODELO TEÓRICO}

Consideremos que la partícula magnética puede ser un mono-dominio magnético, tal que su magnetización es representada por el vector de magnetización $\mathbf{M}=\mathbf{M}(t)$. La evolución temporal de la partícula puede ser modelada por la ecuación vectorial de LLG adimensional [6]:

$$
\frac{d \mathbf{m}}{d \tau}=-\mathbf{m} \times \Gamma-\eta \mathbf{m} \times(\mathbf{m} \times \Gamma),
$$

donde $\mathbf{m}=\mathbf{M} / M_{s}$ y donde $\tau=t|\gamma| M_{s} / \kappa$ con $\kappa=1+\eta^{2}$. Aquí $M_{s}$ es la magnetización de saturación, produciendo que la normalización sea a la unidad $|\mathbf{m}|=1$. El factor giromagnético está representado por $\gamma$, éste está asociado al espín del electrón tal que su valor numérico aproximado está dado por $|\gamma| \approx 2,21 \times 10^{5} m A^{-1} s^{-1}$. Además, $\eta$ denota el coeficiente de disipación fenomenológico adimensional, el cual es una propiedad del material magnético, con valores típicos del orden de $10^{-4} \mathrm{a}$ $10^{-3}$ en granates; y $10^{-2}$ o mayores para cobalto, níquel o en permalloy $\left(\mathrm{Ni}_{80} \mathrm{Fe}_{20}\right)$ [6]. Con estos órdenes de magnitud se tiene que $1 / \kappa \approx 0,99 \approx 1$. Valores experimentales típicos de $M_{s}$ son por ejemplo $M_{s[\mathrm{Co}]} \approx 1,42 \times 10^{6} \mathrm{~A} / \mathrm{m}$ para materiales de cobalto y $M_{s[\mathrm{Ni}]} \approx 4,8 \times 10^{5} \mathrm{~A} / \mathrm{m}$ para materiales de níquel, por lo tanto se tiene que $\mid \gamma / M_{s[\mathrm{Co}]} \approx 308 G H z$ y que $|\gamma|$ $M_{s[\mathrm{Ni}]} \approx 106 \mathrm{GHz}$, respectivamente. Esto implica que las escalas de tiempo para estos materiales son $(|\gamma|$ $\left.M_{s[\mathrm{Co}]}\right)^{-1} \approx 3$ ps y $\left(\mid \gamma / M_{s[\mathrm{Ni}]}\right) \approx 6 p s$, respectivamente.

Además, cabe señalar que en estos materiales la aproximación de macrospin (partículas descritas por mono-dominios magnéticos) es válida para partículas con tamaños aproximados de 10-20 $\mathrm{nm}$, ya que para tamaños menores los efectos de anisotropía de superficie son relevantes [26]; y para 
tamaños mayores aparecen estados magnéticos nouniformes, como por ejemplo vértices en nanohilos de cobalto. Asimismo, la forma de la partícula tiene un rol importante en la aproximación de macrospin [27].

El campo efectivo, $\Gamma$, que actúa sobre la magnetización viene dado por:

$$
\Gamma=\mathbf{h}+\beta(\mathbf{m} \cdot \hat{\mathbf{n}}) \hat{\mathbf{n}},
$$

donde $\mathbf{h}$ es el campo magnético externo y $\beta$ mide la anisotropía a lo largo del eje $\hat{\mathbf{n}}$; el cual en lo que sigue lo tomaremos como el eje z. Esta anisotropía es uni-axial, y la constante $\beta$ depende de la sustancia específica y de la forma de la muestra. De hecho, puede tomar tanto valores positivos como negativos [6]. Nótese que este término proviene de una energía de anisotropía cuadrática, $E_{A N}=-\beta(\mathbf{m} \cdot \hat{\mathbf{n}})^{2}$. El campo magnético externo se asume que está compuesto de dos partes, una constante y otra dependiente de una modulación temporal. En particular, usaremos la siguiente configuración [23]:

$$
\mathbf{h}=h_{z} \hat{\mathbf{z}}+h_{x 1} \sin \left(\Omega_{1} \tau\right) \hat{\mathbf{x}}+h_{x 2} \sin \left(\Omega_{2} \tau\right) \hat{\mathbf{x}},
$$

donde los coeficientes $\left\{h_{z}, h_{x 1}, \Omega_{1}, h_{x 2} \Omega_{2}\right\}$ son constantes. De acorde a la selección de escalamiento dimensional, el campo externo y las frecuencias adimensionales son $\mathbf{h}=\mathbf{H} / M_{s}$ y $\Omega_{j}=\omega_{j} \kappa /\left(\gamma M_{s}\right)$ con $j=(1,2)$. Dado que la amplitud del campo $\mathbf{H}$ $\mathrm{y}$ de las frecuencias son medidas en unidades de $M_{s}$ y de $\left(\gamma M_{s}\right)$, respectivamente, uno encuentra que para materiales como el cobalto o el níquel los órdenes de magnitud son $10^{0}-10^{1} \mathrm{kOe}$ y $\mathrm{GHz}$. En este artículo, se variarán los parámetros en el mismo rango de magnitudes.

Por otra parte, existen dos casos relevantes para la razón entre las frecuencias, $\Omega_{1} / \Omega_{2}$. Cuando $\Omega_{1} / \Omega_{2}$ es racional, el sistema está forzado periódicamente (P), mientras que un valor irracional de $\Omega_{1} / \Omega_{2}$ produce un forzamiento cuasiperiódico $(\mathrm{CP}) \mathrm{de}$ dos-frecuencias.

Finalmente, remarcamos que cuando el sistema tiene un forzamiento $\mathrm{CP}$ el sistema tridimensional no-autónomo (1) puede ser descrito por un sistema autónomo equivalente de dimensión cinco. Como el sistema tiene la restricción de que el módulo de la magnetización es una constante de movimiento con $|\mathbf{m}|=1$, implica que el sistema dinámico autónomo efectivo es de dimensión cuatro.

\section{Caso Conservativo}

Cuando la disipación es nula $(\eta=0)$ y $\sin$ forzamiento paramétrico $\left(h_{x 1}=h_{x 2}=0\right)$ la ecuación vectorial (1) es conservativa, y puede ser derivada desde un funcional de energía. Es decir:

$$
\frac{d \mathbf{m}}{d \tau}=\mathbf{m} \times \nabla_{\mathbf{m}} G(\mathbf{m}),
$$

donde $G$ denota la energía libre. En este caso el campo efectivo se reduce a $\Gamma_{0}=\left(h_{z}+\beta m_{z}\right) \hat{\mathbf{z}}, \mathrm{y}$ por lo tanto las componentes de la ecuación (1) pueden ser escritas como:

$$
\begin{gathered}
\frac{d m_{x}}{d \tau}=-\left(h_{z}+\beta m_{z}\right) m_{y}, \\
\frac{d m_{y}}{d \tau}=-\left(h_{z}+\beta m_{z}\right) m_{x}, \\
\frac{d m_{z}}{d \tau}=0,
\end{gathered}
$$

Este conjunto de ecuaciones tiene una familia de soluciones homoclinas dado por:

$$
\begin{gathered}
m_{x, h}(\tau)=m_{x 0} \cos \left(\Phi_{0} \tau\right)-m_{y 0} \sin \left(\Phi_{0} \tau\right), \\
m_{y, h}(\tau)=m_{y 0} \cos \left(\Phi_{0} \tau\right)-m_{x 0} \sin \left(\Phi_{0} \tau\right), \\
m_{z h}(\tau)=m_{z 0},
\end{gathered}
$$

donde $\Phi_{0}=h_{z}+m \beta_{z 0}$ y el periodo $T_{H O}=2 \pi / \Phi_{0}$. Las condiciones iniciales tienen la restricción dada por el módulo $m_{x 0}^{2}+m_{y 0}^{2}+m_{z 0}^{2}=1$. Para una energía libre fija $G$, se tiene que: $m_{z 0}=\beta^{-1}\left(-h_{z} \pm \sqrt{h_{z}^{2}-2 \beta G}\right)$. Dado que $m_{z 0}$ debe ser real, las soluciones serán estables hasta: $\beta_{c}=h_{z}^{2}(2 G)$.

Por otra parte, la disipación y la inyección de la energía colocan a la partícula en situaciones fuera del equilibrio. Estos fenómenos serán analizados en la próxima sección. 


\section{SIMULACIONES}

Esta sección la dividiremos en dos partes. En la primera sub-sección discutimos brevemente los indicadores con los cuales vamos a caracterizar los diferentes regímenes; $y$ en la segunda son presentados los resultados numéricos y su correspondiente análisis.

\section{Indicadores}

El sistema dinámico descrito por la ecuación (1) bajo la acción de un campo externo dependiente del tiempo de la forma (3) no puede ser resuelto analíticamente, esto es debido a las no-linealidades y las dependencias temporales. Por lo tanto, se debe resolver numéricamente. En orden de integrar numéricamente las ecuaciones de movimiento y abolir artefactos numéricos, es recomendable resolver la ecuación (1) en su representación cartesiana. Asimismo, para obtener una precisión de $10^{-8}$ en el campo de magnetización hemos integrado la ecuación (1) usando el método de Runge-Kutta de cuarto orden con un paso de tiempo fijo $\mathrm{d} \tau=0,01$.

En primer lugar, se hace la caracterización de los comportamientos dinámicos de la ecuación (1) mediante la evaluación de los exponentes de Lyapunov (ELs). Este método consiste en cuantificar la divergencia entre dos trayectorias cercanas del campo vectorial $[1,15,18,25,28]$.

En general, para un sistema dinámico de dimensión efectiva $N$ descrito por un conjunto de ecuaciones de la forma, $d X^{i} / d \tau=F^{i}(\mathbf{X}, \tau)$, el $i$ - ésimo exponente de Lyapunov viene dado por:

$$
\lambda_{i}=\lim _{\tau \rightarrow \infty} \frac{1}{\tau} \ln \left(\frac{\left\|\delta X_{\tau}^{i}\right\|}{\left\|\delta X_{0}^{i}\right\|}\right),
$$

donde $\left\|\delta X_{\xi}^{i}\right\|$ es la distancia entre las trayectorias de la $i$-ésima componente del campo vectorial en el tiempo $\xi$. Estos exponentes pueden ser en forma descendente, desde el mayor hasta el menor: $\lambda_{1} \geq \lambda_{2} \geq$ $\ldots \geq \lambda_{N}$. El primer exponente, $\lambda_{1}$, se conoce como el máximo exponente de Lyapunov (MEL). Explorando la dependencia del MEL con los diferentes parámetros del sistema, uno puede identificar áreas en el espacio de parámetros donde la dinámica es caótica (MEL positivo), o bien donde se presentan dinámicas no-caóticas regulares (MEL cero o negativo). Como estamos tratando con un sistema forzado con dosfrecuencias, al menos dos de los exponentes de Lyapunov serán siempre cero; por lo tanto el atractor más simple será un Toro $T^{2}$ [19]. Otra posibilidad es tener tres exponentes de Lyapunov igual a cero. En este caso el sistema exhibe un comportamiento $\mathrm{CP}$ de tres-frecuencias $T^{3}$. Enfatizamos que un sistema forzado cuasi-periódicamente puede exhibir otros tipos de atractores llamados atractores extraños no-caóticos. Éstos poseen una topología no-suave, pero no tienen una dependencia en la sensibilidad de las condiciones iniciales, que se traduce en tener un MEL no positivo [20]. Los exponentes de Lyapunov son calculados con las técnicas expuestas en las referencias $[15,18,25]$. El error es del orden del $1 \%$, el cual es suficientemente pequeño para el presente análisis.

Además de este método, existen otros indicadores de cómo medir los diferentes estados dinámicos de un sistema, tal como el espectro de Fourier, secciones de Poincaré, funciones de correlación, o diagramas de bifurcación, entre otros $[5,6$, $11,12,15-18]$. El más clásico es el espectro de Fourier, el cual denotaremos por $S(f)$. Usando este espectro uno puede definir la función de distribución de espectral $N(\sigma)$ como el número de cúspides de $/ S(f) /$ con amplitudes mayores que $\sigma$. Esta cantidad obedece diferentes leyes de escala dependiendo del comportamiento dinámico. En particular, $N(\sigma) \propto \ln (1 / \sigma)$ para un comportamiento CP de dos frecuencias, $N(\sigma) \propto(\ln (1 / \sigma))^{2}$ para un comportamiento $\mathrm{CP}$ de tres frecuencias, y $N(\sigma) \propto \sigma^{-\alpha}$ con $1<\alpha<2$, para atractores extraños no-caóticos [19, 22]. En el caso del espectro Fourier para regímenes caóticos, típicamente se observa una gran cantidad de cúspides, los cuales forman como un "continuo" de puntos [5].

El cálculo de la transformada rápida de Fourier es computado después de un lapso suficientemente largo para obtener el estado permanente del sistema, y tomando en consideración lo expuesto en las referencias $[22,28]$.

\section{RESULTADOS}

Los principales resultados están dados en las Figuras 1-9. En particular, centraremos la discusión en la influencia de la componente del campo externo 
dependiente del tiempo. En lo que sigue los valores de los parámetros fijos son: $\eta=0,05, \beta=1$ y $h_{z}=0,1$.

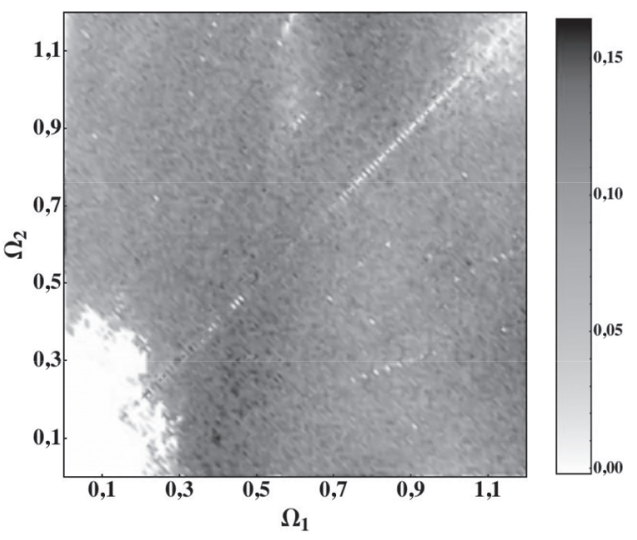

Figura 1. Diagrama de fase que muestra el máximo exponente de Lyapunov en código de color en función de $\Omega_{1}$ y de $\Omega_{2}$. Los valores fijos de la amplitud de campo son $h_{x 1}=1,0$ y $h_{x 2}=0,5$. El diagrama contiene 135939 MELs.

La Figura 1 muestra un diagrama de fase del MEL, $\lambda_{1}$, en función de ambas frecuencias $\Omega_{1}$ y $\Omega_{2}$. Como se puede observar para bajas frecuencias, $\Omega_{1} \leq 0,32$ y $\Omega_{2} \leq 0,28$, existen regiones de dinámica regular $\left(\lambda_{1} \leq 0\right)$. También en la línea $\Omega_{1}=\Omega_{2}$, existen puntos aislados donde la dinámica es cuasiperiódica; en otros casos la dinámica es caótica, ya que $\lambda_{1}>0$. Otras islas cuasiperiódicas aisladas se pueden observar en el diagrama fuera de esta línea. Asimismo, podemos verificar que no hay una simetría de reflexión para $\Omega_{1}=\Omega_{2}$ ya que los valores de las amplitudes son distintas, $h_{x 1} \neq h_{x 2}$.

La Figura 2 muestra un diagrama de fase para el máximo exponente de Lyapunov, $\lambda_{1}$, en función de ambas amplitudes de campo $h_{x 1}$ y $h_{x 2}$, para un forzamiento cuasiperiódico $\Omega_{2} / \Omega_{1}=\pi / 4$. Podemos observar que existe un patrón intrincado de aparición de regiones caóticas y cuasi-periódicas. Este patrón se vuelve más regular cuando la amplitud de $h_{x 1}$ aumenta. El panel ( $a$ ) de la Figura 3 muestra el MEL, $\lambda_{1}$, en función de la amplitud del campo $h_{x 1}$ cuando $h_{x 2}=0,1$ para los mismos parámetros fijos de la Figura 2. Aquí, mostramos un mayor rango de valores de $h_{x 1}$, para estudiar en más detalle el patrón de transición regular-caótico en el espacio de parámetros. La periodicidad de las regiones caóticas cumple con una ley lineal doble, esto es, los

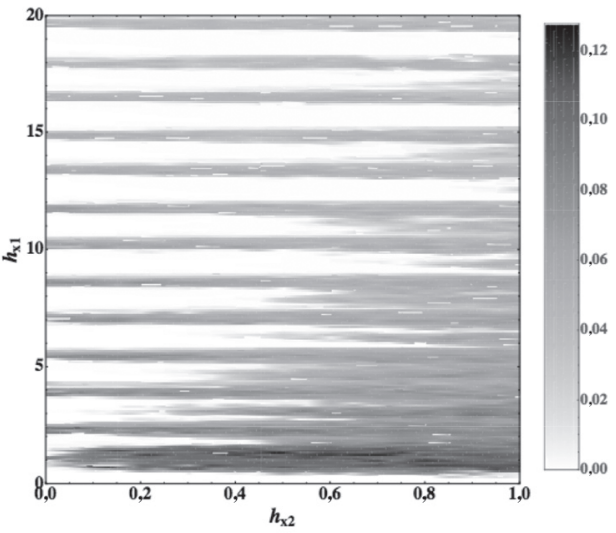

Figura 2. Diagrama de fase que muestra el máximo exponente de Lyapunov en código de color en función de $h_{x 2}$ y de $h_{x 1}$. Los valores fijos de las frecuencias son: $\Omega_{1}=1$ y $\Omega_{2}=\pi / 4$. El diagrama contiene 8421 MELs.

máximos están localizados en $h_{x 1} \sim 0,002+0,09 n$ para $h_{x 1}<40$ donde $n$ indica el número ordinal de máximos y con una doble distancia aproximada $\Delta h_{x 1} \sim 3,1$ para $40 \leq h_{x 1} \leq 100$.

En el panel (b) de la Figura 3 se presenta una comparación entre el exponente de Lyapunov para un forzamiento cuasi-periódico débil $\left(h_{x 2}=0,1\right)$ respecto del periódico $\left(h_{x 2}=0\right)$. En particular, se calcula la diferencia de ambos, $\lambda_{1}\left(h_{x 2}=0,1\right)-\lambda_{1}\left(h_{x 2}=0\right)$, en función de la amplitud del campo $h_{x 1}$.

Además, es interesante hacer notar que para valores mayores de la amplitud, $h_{x 1}>75$, la aparición de regiones caóticas decae drásticamente y para valores $h_{x 1}>100$ las regiones caóticas no son más visibles, y por ende el sistema exhibe estados regulares. Como podemos apreciar, las mayores diferencias son de $10 \%$ para valores bajos e intermedios de campo, mientras que éstos tienden a disminuir considerablemente para altos valores de campo. Ahora bien, aunque estas diferencias no sean por un gran porcentaje son significativas y la topología de los atractores es completamente distinta. Por ejemplo, para valores altos de campo ambos MEL tienden a cero; sin embargo en el caso de forzamiento periódico, el estado regular es un estado periódico, mientras que en el forzamiento cuasiperiódico es un estado cuasiperiódico, el cual puede ser de dos o tres frecuencias dependiendo del valor de campo. 

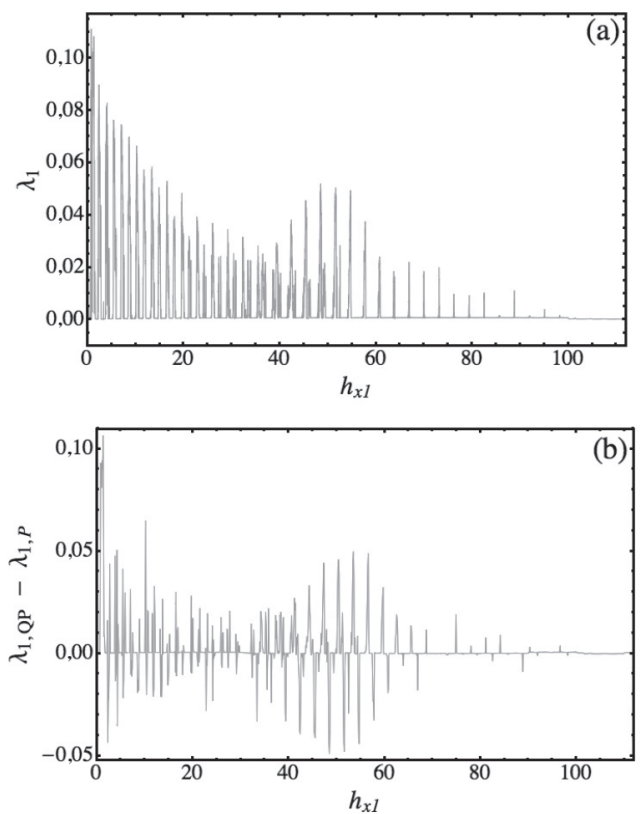

Figura 3. (a) Máximo exponente de Lyapunov en función de $h_{x 1}$ en el caso de forzamiento cuasiperiódico $\left(h_{x 2}=0,1\right)$ (b) Diferencia entre el máximo exponente Lyapunov del cuasiperiódico y el periódico $\left(h_{x 2}=0\right)$ en función de $h_{x 1}$. Los valores fijos de frecuencias son: $\Omega_{1}=1, \Omega_{2}=\pi / 4$. La resolución es $\delta h_{x 1}=0,01$.

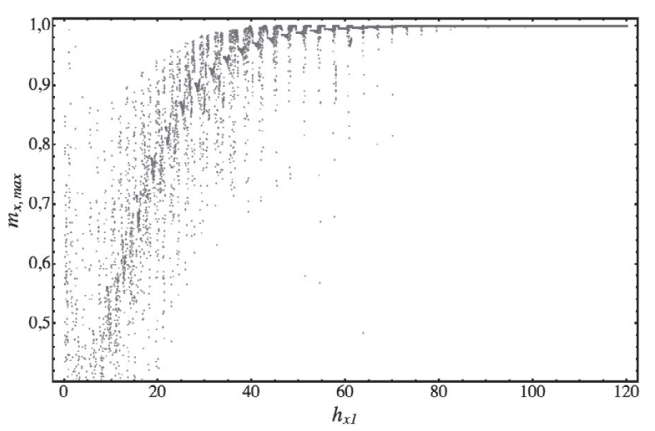

Figura 4. Diagrama de bifurcación de $m_{x}$ en función de $h_{x 1}$. Los parámetros fijos son los mismos que en la Figura 3(a).

En la Figura 4 se expone el diagrama de bifurcación de la componente $x$ de la magnetización $m_{x}$, en función de $h_{x 1}$. Este diagrama es calculado tomando en consideración los máximos locales de la serie temporal de la componente en cada valor de campo.
Como podemos ver las regiones caóticas tienen una dispersión amplia mientras que en los estados no-caóticos la dispersión está condensada. Además, se corrobora el hecho de que para valores altos de campo el sistema tiende a estados regulares.

En orden de particularizar los diferentes tipos de comportamientos que exhibe el sistema, a continuación se muestran casos selectos de valores de campo.

Las Figuras 5 y 6 muestran la serie temporal de la componente $x$ de la magnetización, $m_{x}$, una sección de Poincaré bidimensional en función de $m_{x}$ y $m_{y}$ obtenido con intervalos temporales de $2 \pi / \Omega_{1}$, el espectro de Fourier de $m_{x}$ y su correspondiente función de distribución espectral, $N(\sigma)$, para dos casos particulares de campo $h_{x 1}=0,02$ y $h_{x 1}=1,84$, respectivamente. El resto de los parámetros fijos son los mismos que en la Figura 3. Cabe destacar que en ambos casos el MEL, $\lambda_{1}$, es nulo desde el punto de vista numérico, ya que sus valores están por debajo de $10^{-4}$, el cual es considerado como cero numérico para el computo de los exponentes de Lyapunov. Por lo tanto, el sistema se encuentra en unos regímenes no-caóticos.

Como se puede apreciar en los paneles (a) de ambas figuras la línea punteada siempre se mantiene en la unidad. Esto es consecuente con la teoría, ya que se ha normalizado a la unidad, esto es $/ \mathrm{m} /=1$. Conjuntamente, podemos observar que las series temporales no son abruptas ni irregulares.

Por otra parte, los paneles (b) muestran las secciones de Poincaré comprobándose que son curvas cerradas y suaves, lo que indica que estamos en regímenes cuasiperiódicos. Para poder saber qué tipo de cuasiperiodicidad se presenta recurrimos al espectro de Fourier que se muestra en los paneles (c) de cada figura, éstos muestran un conjunto de picos discretos.

En particular, en el caso de la Figura 5 aparecen bastante pocos, habiendo tan solo dos picos dominantes.

En los paneles (d) de las Figuras 5 y 6 se observa la función de distribución espectral $N(\sigma)$, la cual nos proporciona una caracterización más depurada. De hecho, para el caso de $h_{x 1}=0,02$ (Figura 5) 

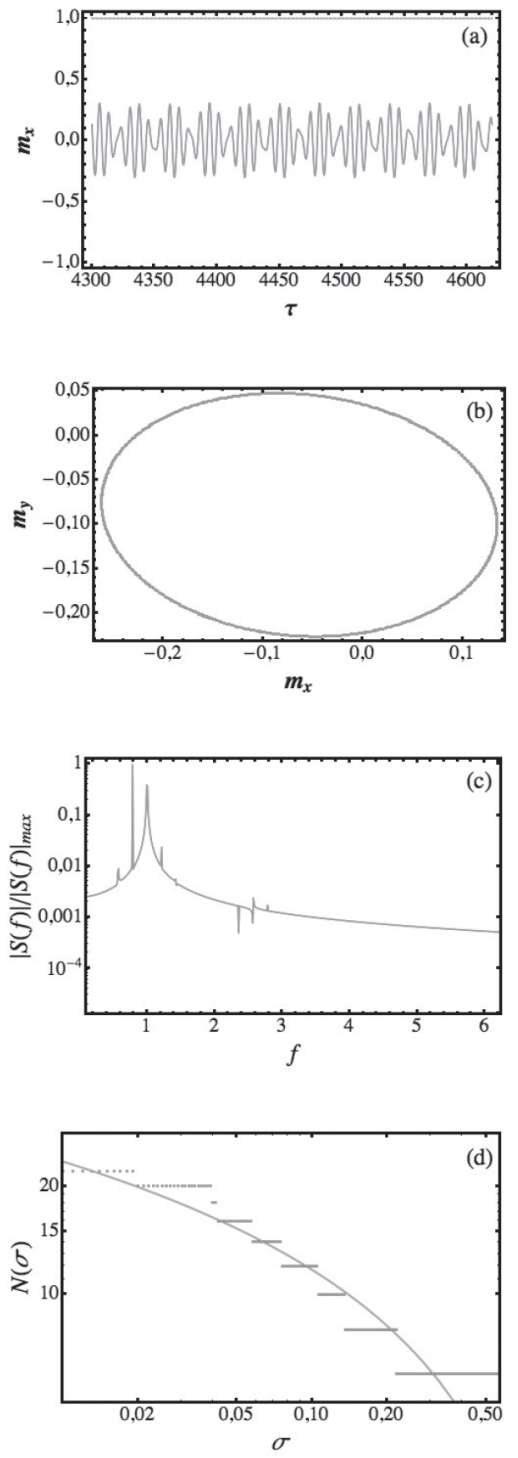

Figura 5. (a) $m_{x}$ (línea continua) y $/ \mathbf{m} /$ (línea punteada) en función del tiempo $\tau$. (b) Sección de Poincaré de $m_{x}-m_{y}$ en intervalos de tiempo de $2 \pi / \Omega_{1}$. (c) Espectro de Fourier normalizado, $|S(f)| /|S(f)|_{\max }$, en función de la frecuencia $f$. (d) Función de distribución espectral $N(\sigma)$ en función de $\sigma$. El valor de campo es $h_{x 1}=0,02$, y el resto de parámetros fijos son los mismos que en la Figura 3(a).

encontramos que $N(\sigma)=\mu \ln (1 / \sigma)$ con $\mu \approx 5,174$. Esto implica que el sistema está en un régimen cuasiperiódico de dos frecuencias. Por otra parte, en el caso de $h_{x 1}=1,84$ (Figura 6) se tiene que la
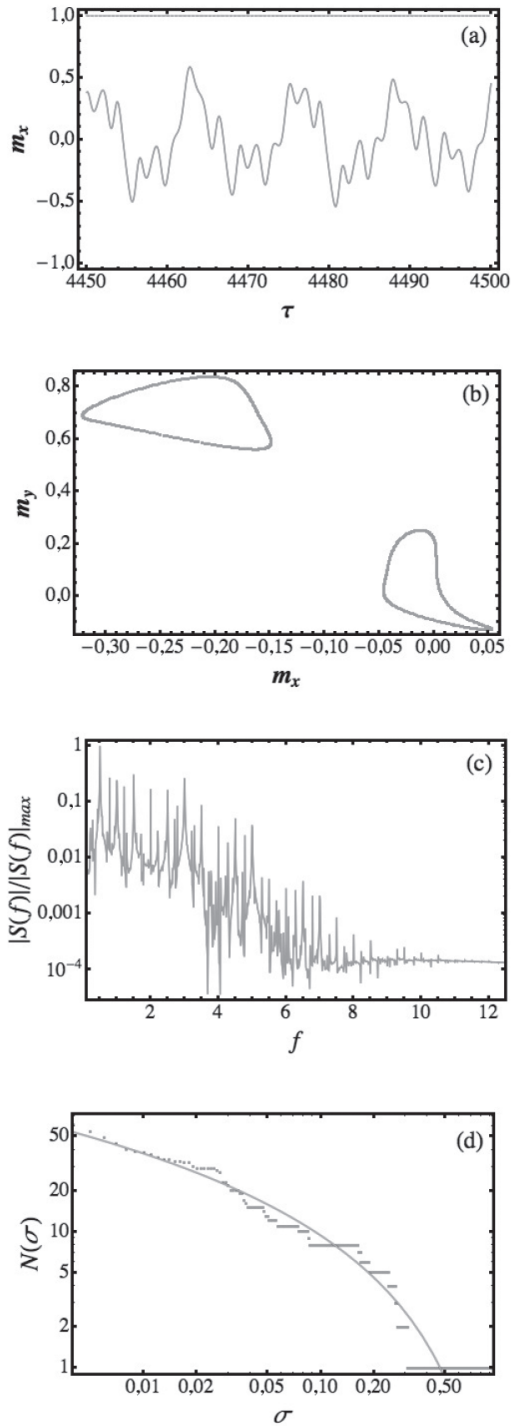

Figura 6. (a) $m_{x}$ (línea continua) y / m / (línea punteada) en función del tiempo $\tau$. (b) Sección de Poincaré de $m_{x}-m_{y}$ en intervalos de tiempo de $2 \pi / \Omega_{1}$. (c) Espectro de Fourier normalizado, $|S(f)| /|S(f)|_{\max }$, en función de la frecuencia $f$. (d) Función de distribución espectral $N(\sigma)$ en función de $\sigma$. El valor de campo es $h_{x 1}=1,84$ y el resto de parámetros fijos son los mismos que en la Figura 3(a).

distribución espectral ahora ajusta acorde a la ley $N(\sigma)=\mu \ln (1 / \sigma)^{2}$ con $\mu \approx 1,767$. Luego, para estos parámetros el sistema exhibe un comportamiento cuasiperiódico de tres frecuencias. 
En la Figura 7 se muestran los mismos paneles que en las dos figuras precedentes para un valor de campo $h_{x 1}=16,50$. En este caso el sistema se encuentra en un estado caótico ya que el valor del MEL es $\lambda_{1}=0,049$. Como podemos observar en el panel (a), la serie temporal es irregular. Claramente, la sección de Poincaré no es una curva cerrada, y más aún es rugosa como se puede apreciar en el panel (b). Como se muestra en el panel (c), la transformada de Fourier muestra un continuo de puntos, tal que su distribución espectral puede ser ajustada con una ley potencial $N(\sigma)=\mu \sigma^{-\alpha}$, donde $\mu \approx 0,765$ y donde $\alpha \approx 0,935$.

Un caso de atractor extraño no caótico se presenta en la Figura 8. Este atractor se encuentra para un valor de $h_{x 1}=26,41$. En este caso el valor del máximo exponente de Lyapunov es nulo desde el punto de vista numérico, de hecho éste es $\lambda_{1}=4 \times 10^{-5}$, lo cual está muy por debajo del valor de corte. Los paneles contienen los mismos indicadores que las tres figuras anteriores. La serie temporal muestra rasgos de irregularidad tanto en los valores máximos como mínimos. Como se puede apreciar claramente en el panel (b) el mapa temporal de Poincaré es el de un atractor extraño.

De hecho, la ley que ajusta la función de distribución espectral es $N(\sigma)=\mu \sigma^{-\alpha}$, con $\mu \approx 0,6602$ y $\alpha \approx 1,00767$, lo cual es consecuente con la teoría.

Cabe destacar que este tipo de atractores son difíciles de obtener ya que los métodos directos de cómputo como los exponentes de Lyapunov, no generan información de ellos, y se tiene que recurrir a otros métodos como el ajuste de la función espectral entre otros [19-23].

Remarcamos también que este tipo de regímenes son sólo obtenidos con forzamientos cuasiperiódicos. Por otra parte, en este problema magnético estos atractores extraños no son muy ocurrentes en el espacio de parámetros, es decir anteceden o preceden al caos en regiones muy acotadas del espacio de parámetros.

Finalmente, en la Figura 9 se muestran dos secciones tridimensionales de Poincaré obtenidas con intervalos temporales de $2 \pi / \Omega_{1}$ cuando el sistema exhibe un atractor extraño no caótico. Estos dos casos particulares se encuentran para los
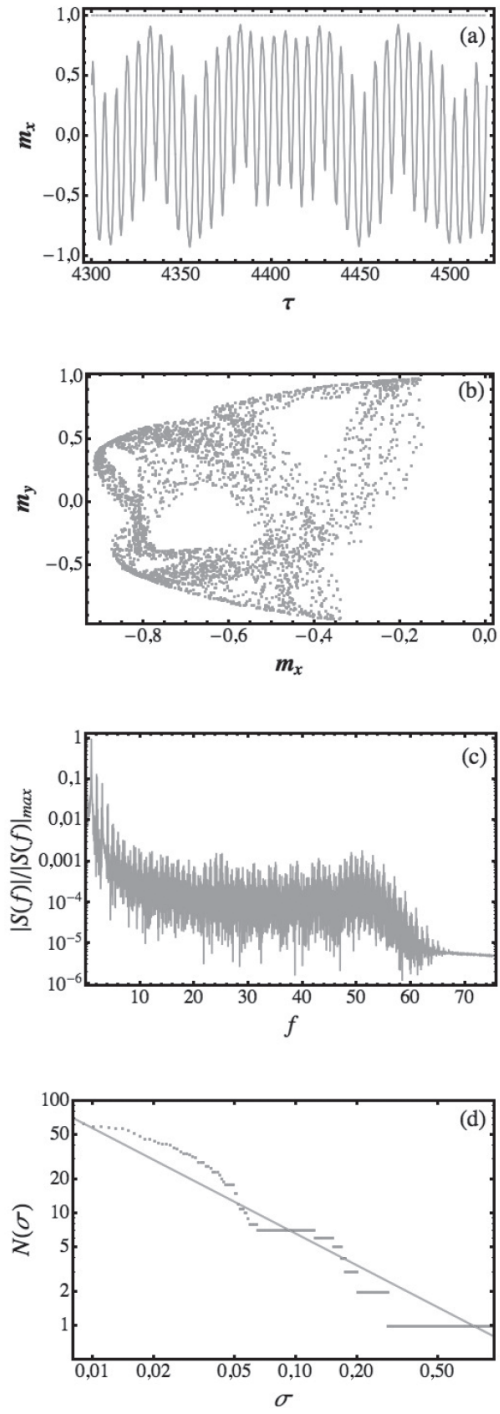

Figura 7. (a) $m_{x}$ (línea continua) y / m / (línea punteada) en función del tiempo $\tau$. (b) Sección de Poincaré de $m_{x}-m_{y}$ en intervalos de tiempo de $2 \pi / \Omega_{1}$. (c) Espectro de Fourier normalizado, $|\mathrm{s}(f)| /|\mathrm{s}(f)|_{\max }$ en función de la frecuencia $f$. (d) Función de distribución espectral $N(\sigma)$ en función de $\sigma$. El valor de campo es $h_{x 1}=16,50$ y el resto de parámetros fijos son los mismos que en la Figura 3(a).

valores de campo $h_{x 1}=24,39$ y $h_{x 1}=27,64$. Como se puede ver en ambos casos el mapa es rugoso y con discontinuidades, siendo una característica de estos atractores. Obviamente, los valores del MEL son no positivos para ambos casos. 

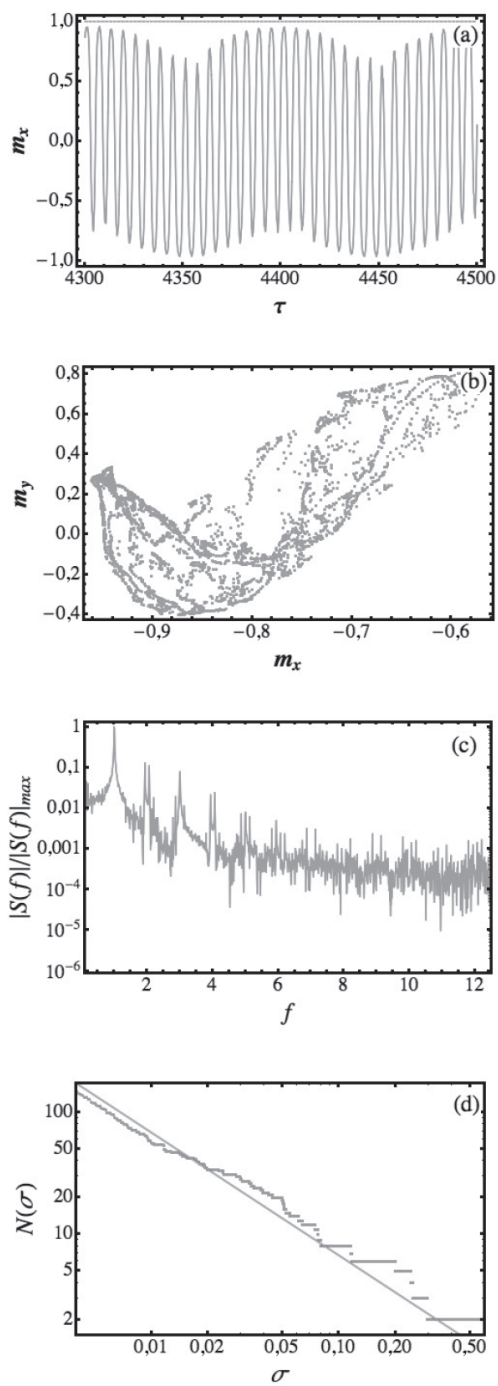

Figura 8. (a) $m_{x}$ (línea continua) y $/ \mathbf{m} /$ (línea punteada) en función del tiempo $\tau$. (b) Sección de Poincaré de $m_{x}-m_{y}$ en intervalos de tiempo de $2 \pi / \Omega_{1}$. (c) Espectro de Fourier normalizado, $|S(f)| /|S(f)|_{\max }$, en función de la frecuencia $f$. (d) Función de distribución espectral $N(\sigma)$ en función de $\sigma$. El valor de campo es $h_{x 1}=26,41$ y el resto de parámetros fijos son los mismos que en la Figura 3(a).

\section{CONCLUSIONES}

En el presente artículo se ha estudiado la dinámica de la magnetización de una partícula magnética con anisotropía cuadrática uni-axial descrita por
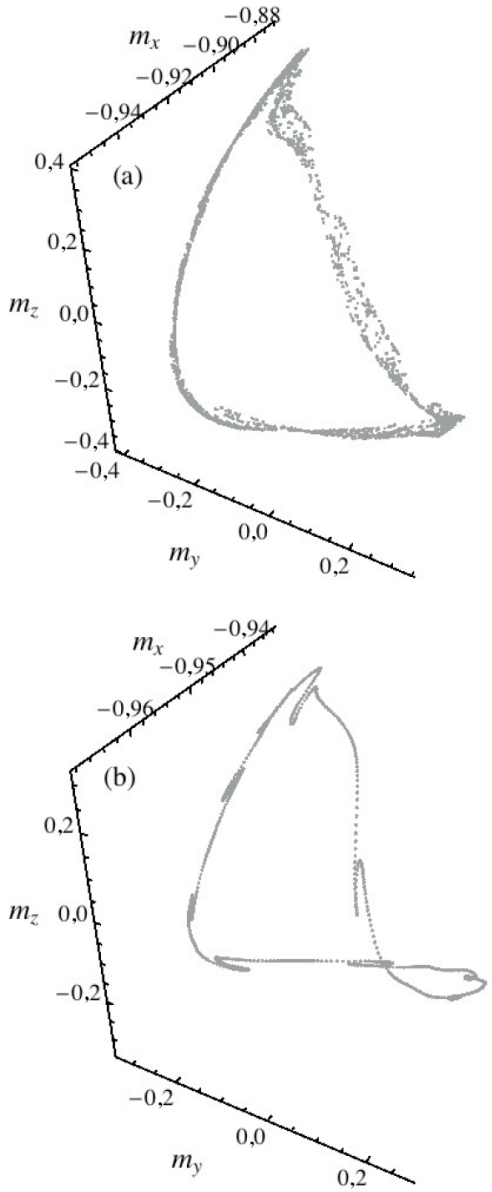

Figura 9. Sección de Poincaré de $\mathbf{m}$ en intervalos de tiempo de $2 \pi / \Omega_{1}$. Los valores de campo $h_{x 1}=24,39$ y $h_{x 1}=27,64$. El resto de parámetros fijos son los mismos que en la Figura 3 (a).

la ecuación de LLG. Particularmente, nos hemos enfocado en comprender el efecto de forzamientos tanto periódicos como cuasiperiódicos en el tiempo, extendiendo los resultados obtenidos en la literatura sobre este tópico [11, 15-18, 23]. Para caracterizar la dinámica, hemos empleado distintos métodos, como transformada de Fourier, diagramas de bifurcaciones y exponentes de Lyapunov. En primer lugar, hemos calculado el máximo exponente de Lyapunov en función de ambas frecuencias, lo cual da regiones de ambos tipos de forzamiento dependiendo de la razón entre las frecuencias, $\Omega_{1} / \Omega_{2}$. En la línea $\Omega_{1}=\Omega_{2}$, que da un forzamiento periódico, hemos encontrado que para frecuencias intermedias y altas existen islas de comportamientos dinámicos 
regulares $\lambda \leq 0$. Luego, nos hemos centrado en el caso de forzamientos cuasiperiódicos con una relación particular de $\Omega_{1} / \Omega_{2}=4 / \pi$. En este caso, hemos variado simultáneamente ambas amplitudes de campos de forzamiento $h_{x 1}$ y $h_{x 2}$. Se ha encontrado un patrón intrincado de transición entre estados caóticos y no caóticos. Para poder analizar en más detalle las transiciones hemos escogido un valor particular del campo asociado a la segunda frecuencia de forzamiento dado por $h_{x 2}=0,1 \mathrm{y}$ logramos deducir cómo es la periodicidad de la aparición del caos. Además, se comparó con el caso de forzamiento puramente periódico encontrando diferencias de hasta un $10 \%$ en la amplitud del máximo exponente de Lyapunov a lo largo de la línea $h_{x 1}$.

Remarcamos nuevamente que a pesar de la poca diferencia en las amplitudes los estados no caóticos tienen una topología muy diferente, ya que en el caso cuasiperiódico el estado más simple es un Toro. Posteriormente, analizamos casos particulares sobre la línea $h_{x 1}$, obteniendo tanto estados cuasiperiódicos de dos y tres frecuencias caóticos, como también atractores extraños no caóticos. Estos últimos estados preceden o anteceden al caos en regiones muy acotadas del espacio de parámetros. Finalmente, destacamos que como los parámetros escogidos están dentro del rango experimental creemos que estos estados podrían ser medidos.

\section{AGRADECIMIENTOS}

Los autores agradecen invaluables discusiones con J.A.C. Gallas (Universidade do Paraíba, Brasil), H. Pleiner (Max-Planck-Institute für Polymerforschung, Alemania), y con D. BecerraAlonso (Universidad Loyola Andalucía, España). Este trabajo de investigación cuenta con el apoyo financiero otorgado por los proyectos FONDECYT 1120764, CEDENNA, Millennium Scientific Initiative P10-061F, y UTA Project 8750-12.

\section{REFERENCIAS}

[1] S.A. Wolf, D.D. Awschalom, R.A. Buhrman, J.M. Daughton, S. von Molnar, M.L. Roukes, A.Y. Chtchelkanova and D.M. Treger. "Spintronics: A Spin-Based Electronics Vision for the Future". Science. Vol. 294, pp. 1488-1495. 2001.
[2] D. Gatteschi, R. Sessoli and J. Villain. "Molecular Nanomagnets". Oxford University Press. UK. 2006.

[3] C. Alexiou, W. Arnold, R.J. Klein, F.G. Parak, P. Hulin, C. Bergemann, W. Erhardt, S. Wagenpfeil and A.S. Lubbe. "Locoregional cancer treatment with magnetic drug targeting". Cancer Res. Vol. 60, Issue 23, pp. 6641. 2000.

[4] V.V. Kruglyak, S.O. Demokritov and D. Grundler. "Magnonics". J. Phys. D: Appl. Phys. Vol. 43 264001. 2010.

[5] P.E. Wigen (Ed.). "Nonlinear Phenomena and Chaos in Magnetic Materials". World Scientific. Singapore. 1994.

[6] I.D. Mayergoyz, G. Bertotti, and C. Serpico. "Nonlinear Magnetization Dynamics in Nanosystems". Elsevier. North Holland. 2009.

[7] G. Gibson and C. Jeffries. "Observation of period doubling and chaos in spin-wave instabilities in yttrium iron garnet". Phys. Rev. A. Vol. 29, pp. 811-818. 1984.

[8] F.M. de Aguiar, A. Azevedo and S.M. Rezende. "Characterization of strange attractors in spin-wave chaos". Phys. Rev. B. Vol. 39, pp. 9448-9452. 1989.

[9] J. Becker, F. Rodelsperger, Th. Weyrauch, H. Benner, W. Just and A. Cenys. "Intermittency in spin-wave instabilities". Phys. Rev. E. Vol. 59, pp. 1622-1632. 1999.

[10] J. Cai, Y. Kato, A. Ogawa, Y. Harada, M. Chiba and T. Hirata. "Chaotic dynamics during slow relaxation process in magnon systems". J. Phys. Soc. Jap. Vol. 71, pp. 30873091. 2002.

[11] D.V. Vagin and P. Polyakov. "Control of chaotic and deterministic magnetization dynamics regimes by means of sample shape varying”. J. App. Phys. Vol. 105, 033914. 2009.

[12] D. Laroze and P. Vargas. "Dynamical behavior of two interacting magnetic nanoparticles". Phys. B. Vol. 372, pp. 332-336. 2006.

[13] D. Laroze and L.M. Pérez. "Classical spin dynamics of four interacting magnetic particles on a ring". Physica B. Vol. 403, pp. 473-477. 2008.

[14] D. Laroze, P. Vargas, C. Cortes and G. Gutiérrez. "Dynamics of two interacting dipoles". Journal of Magnetism and Magnetic Materials. Vol. 320, pp. 1440-1448. 2008. 
[15] L.F. Álvarez, O. Pla and O. Chubykalo. "Quasiperiodicity, bistability, and chaos in the Landau-Lifshitz equation". Phys. Rev. B. Vol. 61, pp. 11613-11617. 2000.

[16] R.K. Smith, M. Grabowski and R.E. Camley. "Period doubling toward chaos in a driven magnetic macrospin". J. Magn. Magn. Mater. Vol. 322, pp. 2127-2134. 2010.

[17] D. Laroze, O. J. Suárez, J. Bragard and H. Pleiner. "Characterization of the Chaotic Magnetic Particle Dynamics". IEEE Trans. On Magnetics. Vol. 47, pp. 3032-3039. 2011.

[18] J. Bragard, H. Pleiner, O.J. Suárez, P. Vargas, J.A.C. Gallas and D. Laroze. "Chaotic dynamics of a magnetic nanoparticle". Phys. Rev. E. Vol. 84, 037202. 2011.

[19] A. Bondeson, E. Ott and T.M. Antonsen Jr. "Quasiperiodically Forced Damped Pendula and Schrödinger Equations with Quasiperiodic Potentials: Implications of Their Equivalence". Phys. Rev. Lett. Vol. 55, pp. 2103-2106. 1985.

[20] T. Kapitaniak and J. Wojewoda. "Attractors of Quasiperiodically Forced Systems". World Scientific. Singapore. 1994.

[21] M. Agrawal, A. Prasad and Ram Ramaswamy. "Quasiperiodic forcing of coupled chaotic systems". Phys. Rev. E. Vol. 81, 026202. 2010.
[22] W.L. Ditto, M.L. Spano, H.T. Savage, S.N Rauseo, J. Heagy and E. Otth. "Experimental Observation of a Strange Nonchaotic Attractor". Phys. Rev. Lett. Vol. 65, pp. 533536. 1990.

[23] D. Laroze, D. Becerra-Alonso, J.A.C. Gallas and $\mathrm{H}$. Pleiner. "Magnetization dynamics under a quasiperiodic magnetic field". IEEE Transactions on Magnetics Vol. 48, pp. 3567-3570. 2012.

[24] U. Feudel, S. Kuznetsov and A. Pikovsky. "Strange non-chaotic attractors". World Scientific. Singapore. 2006.

[25] JAC. Gallas. "The structure of infinite periodic and chaotic hub cascades in phase diagrams of simple autonomous flows". Int. J. Bifur. Chaos. Vol. 20, pp. 197-211. 2010.

[26] X. Batlle and A. Labarta. "Finite-Size Effects In Fine Particles: Magnetic and Transport Properties". J. Phys. D. Vol. 35, pp. R15-R42. 2002.

[27] P. Landeros, J. Escrig, D. Altbir, D. Laroze, J. D'Albuquerque e Castro and P. Vargas. "Scaling relations for magnetic nanoparticles". Phys. Rev. B. Vol. 71, 094435. 2005.

[28] H. Gould and J. Tobochnik "An Introduction to Computer Simulation Methods: Applications to Physical Systems". 2nd edition. AddisonWesley. New York, USA. 1996. 